\section{Maschinelle Beatmung: Beurteilung einer Zwerchfellatrophie}

Zambon $\mathrm{M}$ et al. Mechanical Ventilation and Diaphragmatic Atrophy in Critically III Patients: An Ultrasound Study. Crit Care Med 2016; 44 : $1347-1352$

Eine maschinelle Beatmung trägt zu einer zunehmenden Atrophie der Atemmuskulatur und damit auch des Zwerchfells bei, der sog. Ventilatorinduced Diaphragmatic Dysfunction (VIDD). Diese wiederum kann auch nach Stabilisierung des Patienten die Entwöhnung vom Gerät erschweren und die Beatmungsdauer verlängern. Die VIDD lässt sich aber bislang nur schwer messen - italienische Mediziner stellen nun eine Möglichkeit vor.

Mithilfe sonografischer Messungen kann die Atrophie des Zwerchfells unter maschineller Beatmung quantifiziert werden, sodass sich Risikofaktoren für Entwicklung und Ausmaß einer VIDD identifizieren lassen. Dieses Ergebnis haben Massimo Zambon und seine Kollegen erhalten, die insgesamt 40 Patienten der Universitätsklinik Mailand in ihre prospektive Beobachtungsstudie aufgenommen haben.

Die Teilnehmer waren seit max. 12 Stunden intubiert und wurden mit einer lungenprotektiven Strategie beatmet. Bei ihnen wurde nach Aufnahme in die Studie täglich mittels Ultraschall mit einem Linearschallkopf (Frequenz 7-12 MHz) über ein Schallfenster zwischen dem 8 . und 10. Interkostalraum zwischen vorderer und mittlerer Axillarlinie $0,5-2 \mathrm{~cm}$ unterhalb des Sinus phrenicocostalis die Dicke des Zwerchfells bestimmt. Die Messung erfolgte dabei am Ende der Exspiration, wenn die Dicke ihren Minimalwert erreicht $\left(T_{d i}\right)$.

Als primärer Endpunkt wurde die Veränderung der Zwerchfelldicke unter der maschinellen Beatmung prozentual zu der jeweils vorangegangenen Messung bestimmt $\left(\Delta \mathrm{T}_{\mathrm{di}}\right)$ und zu den Beatmungseinstellungen der letzten $24 \mathrm{~h}$ in Beziehung gesetzt. Dabei unterschieden die
Mediziner 4 Muster der Atemunterstüt-

zung:

- Muster 1: keine Druckunterstützung (Spontanatmung oder CPAP)

- Muster 2: geringe Druckunterstützung $(5-12 \mathrm{~cm} \mathrm{H}$ O)

- Muster 3: hohe Druckunterstützung (>12 $\mathrm{cm} \mathrm{H} \mathrm{H}_{2} \mathrm{O}$ ) und

- Muster 4: vollständig kontrollierte maschinelle Beatmung

Die Auswertung von insgesamt 153 Untersuchungen bei den 40 Patienten zeigte eine durchschnittliche Veränderung der Zwerchfelldicke um:

- $-7,5 \% / \operatorname{Tag}( \pm 12,3 \%)$ bei Beatmungsmuster 4

- $-5,3 \% / \operatorname{Tag}( \pm 12,9 \%)$ bei Muster 3

- $-1,5 \% / T a g( \pm 10,9 \%)$ bei Muster 2 und

- $+2,3 \% / \operatorname{Tag}( \pm 9,5 \%)$ bei Muster 1

In der multivariaten Analyse, in die u. a. Ausmaß der Sedierung, neuromuskuläre Blockade, Tage mit maschineller Beatmung und die genannten Beatmungsmuster eingingen, erwies sich nur das Muster der Atemunterstützung als signifikant mit $\Delta \mathrm{T}_{\mathrm{di}}$ verbunden.

FAZIT

Bei Patienten mit (maschineller) Atemunterstützung findet sich ein linearer Zusammenhang zwischen Invasivität der Beatmung und Zwerchfellatrophie, fassen die Autoren zusammen. Diese Untersuchung zeigt weiterhin die Durchführbarkeit der täglichen sonografischen Messung und ihren Nutzen, um eine sich entwickelnde Zwerchfellatrophie im Verlauf zu überwachen und möglicherweise mithilfe veränderter Beatmungseinstellungen zu minimieren. Einschränkend gilt die geringe Patientenzahl, aufgrund derer weitere ggf. zu einer Atrophie beitragende Faktoren nicht ausreichend sicher identifiziert werden konnten.

Dr. med. Elke Ruchalla, Bad Dürrheim 